Published in final edited form as:

World J Urol. 2020 November ; 38(11): 2987-2994. doi:10.1007/s00345-020-03101-6.

\title{
Challenges facing the urologist in low- and middle-income countries
}

\author{
Ian Metzler ${ }^{1,2}$, David Bayne ${ }^{2}$, Helena Chang ${ }^{1}$, Mohamed Jalloh ${ }^{3}$, Ira Sharlip ${ }^{2}$ \\ ${ }^{1}$ Department of Urology, University of Washington, Seattle, USA \\ ${ }^{2}$ Department of Urology, Parnassus Campus, University of California San Francisco, 400 \\ Parnassus Avenue, UCSF Box 0738, San Francisco, CA, 94143-073 USA \\ ${ }^{3}$ Hopital General Grand Yoff, Dakar, Senegal
}

\begin{abstract}
Purpose-The challenges in providing urologic care across borders and in resource-constrained settings are poorly understood. We sought to better characterize the impediments to the delivery of urological care in low- and middle-income countries (LMICs) compared to high-income countries (HICs).

Methods-A 70 question online survey in RedCap ${ }^{\mathrm{TM}}$ was distributed to urologists who had practiced in countries outside of the United States and Europe categorized by World Bank income groups.
\end{abstract}

\begin{abstract}
Results-114 urologists from 27 countries completed the survey; 35 (39\%) practiced in HICs while $54(61 \%)$ practiced in LMICs. Forty-three percent of urologists received training outside their home country. Most commonly treated conditions were urolithiasis (30\%), BPH (15\%) and prostate cancer (13\%) which did not vary by group. Only $19 \%$ of urologists in LMICs reported sufficient urologists in their country. Patients in LMICs were less likely to get urgent drainage for infected obstructing kidney stones or endoscopic treatment for a painful kidney stone or obstructing prostate. Urologists visiting LMICs were more likely to cite deficits in knowledge, inadequate operative facilities and limited access to disposables as the major challenges whereas local LMIC urologists were more likely to cite financial challenges, limited access to diagnostics and support staff as the barriers to care.
\end{abstract}

Conclusions-LMICs lack enough training opportunities and urologists to care for their population. There is disconnect between the needs identified by local and visiting urologists.

imetzler@uw.edu.

Author contributions

IM: Protocol/project development, manuscript writing. DB: Data analysis, Protocol/project development. MJ: Data collection, manuscript editing. HC: Protocol/project development. IS: Data collection, manuscript editing. All authors contributed to the study conception and design. Material preparation, data collection and analysis were performed by IM and DB. The first draft of the manuscript was written by IM and all authors commented on previous versions of the manuscript.

All authors read and approved the final manuscript.

Compliance with ethical standards

Ethical statement The authors have no disclosures or conflict of interest related to the submitted material. Investigational Review Board designation for this study as exempt was obtained at University of California, San Francisco. 
International collaborations should target broader interventions in LMICs to address local priorities such as diagnostic studies, support staff and financial support.

\section{Keywords}

International; Global; Urology; Low-income countries; Disparities; Access; Socioeconomic; Barriers to care

\section{Introduction}

Surgical services have been shown to be an essential and cost-effective component of health care even in low-resource settings [1]. Dr. Jim Kim, former prior World Bank president, described "surgery is an indivisible, indispensable part of health care and of progress towards universal health coverage." Urology is an essential component of any basic surgical service but the specialty has fallen behind other global surgery efforts [2]. The 2010 Global Burden of Disease study revealed that urologic cancers accounted for over 10 million disability-adjusted life years (DALYs) and benign conditions such as urolithiasis and BPH accounted for 13.5 million DALYs [3]. The burden of these later-in-life diseases has increased by $28 \%$ since the 1990 s as many other conditions that result in early mortality have decreased. These figures may be an underestimation as household surveys have demonstrated a high prevalence of untreated gross hematuria and urinary retention [4]. There is a paucity of publications related to global urologic care performed through international collaboration despite its increasing prevalence and the potential benefits for trainees [5, 6].

There is growing interest in international volunteerism amongst urologists from high-income countries (HICs) and there are several urology-specific organizations that strive to channel this energy into productive, ethical and sustainable programs. IVUmed (Salt Lake City, UT) has been in operation over 23 years and has educated providers and served patients in over 30 countries [7]. Urolink with the British Association of Urological Surgeons (BAUS) has been organizing international workshops to improve urologic care [8]. The Global Philanthropic Committee of the American Urologic Association (AUA), European Urology Association (EAU) and Société Internationale d'Urologie (SIU) have financed many efforts to improve urologic care in LMICs. These organizations have successfully created workshops to train local urologists on subspecialized operations and care. Success is dependent on engaging local stakeholders and eliciting local needs [7]. But international organizations and their local partners face unique challenges in providing care in the varied environments of low- and middle-income countries (LMICs). The needs of urologists working in LMICs are diverse and can be unclear to the visiting urologists and foreign organizations. The purpose of this study was to directly survey urologists who work in LMICs to better understand their background, care-patterns and the challenges they face to better inform international collaboration to improve urologic care worldwide. 


\section{Methods}

\section{Survey design and distribution}

An online-only survey was developed in RedCap ${ }^{\mathrm{TM}}$ with 70 discrete items written in English. The survey was adaptive and length varied depending on the information provided by the respondent. Investigational Review Board designation for this study as exempt was obtained at University of California, San Francisco. Urologists who had experience practicing in countries outside of the United States and Europe were targeted for responses. Surveys were distributed using personal contact by the authors, social networking sites and letters to regional urologic societies and international urologic non-governmental organizations. Country of practice was self-assigned by the respondent and categorized by World Bank income groups: high-income countries (HICs) and low and middle-income countries (LMICs). Survey responses were anonymous unless optional contact information was provided. Practice in another country was defined as spending more than one week practicing urology in a country in the last 5 years. No verification of licensure or certification was performed. Respondents were then categorized into three groups: (1) HICs -urologists that only practiced in HICs, (2) local LMICs-urologists that only practiced in LMICs, and (3) visiting LMICs - urologists that practiced in a secondary country that is designated a LMIC. Respondents who reported practicing as visitors to LMICs recorded responses for their primary country (HIC or LMIC) and the most challenged LMIC country in which they had practiced in the last 5 years.

Most common urologic condition treated were free text entries that were categorized for analysis and the treatments were multiple choice. Questions for clinical inquiry were chosen to represent different specialties for which there were AUA guidelines with the highest rating as a "standard" of care, these included: draining an infected obstructing ureteral stone, offering BCG to patients with high-risk bladder cancer, offering a 24-h urine collection for recurrent stone formers and offering a partial nephrectomy to a patient with a $5 \mathrm{~cm}$ renal mass. Challenge categories were developed in consultation with multiple urologists who have practiced in both HIC and LMIC settings.

Responses were compared between HIC urologists that only practiced in HICs and local LMIC urologists that only practiced in LMICs. We also compared responses between LMIC urologists that only practiced in LMICs and visiting LMIC urologists. Analysis was performed using Student's $t$ test for linear outcomes and fisher's exact tests for binary outcomes to assess for statistical significance in R Version 3.3.3.

\section{Results \\ Demographics}

One-hundred fourteen urologists from 27 countries completed the survey. The mean age of respondents was 47.4 years. Eleven percent were female. Age and gender did not vary by practice group (Table 1). Respondents had been in practice for an average of 17.6 years, including their urology training. Thirty-five (31\%) practiced in HICs only, 54 (47\%) practiced in a single LMIC and 25 (22\%) practiced as a visitor to an LMIC. See Fig. 1 for map of countries with respondents and country of travel. HIC urologists that left to visit 
LMICs were similar in age but were more likely to have been born in an LMIC than HIC urologists that did not leave their country. Forty-four percent of visiting LMIC urologists were born in an LMIC compared to $14 \%$ of HIC urologists that did not leave their country. The majority of survey respondents in both HIC and LMICs practiced at academic referral hospitals in large urban centers ( $>1$ million population) (Table 2). Only $20 \%$ of urologists practicing in LMICs reported that there were enough urologists to treat the population in their country compared to $69 \%$ of urologists in HICs. Fig 2 . shows the travel pattern of urologists going from their home country to visit a LMIC to practice.

The average number of years of urology training was 4.6 years in addition to 2.2 years of general surgery training. Total years of urology training were higher in the HIC urologist group $(p<0.05)$. Forty-two percent of all surveyed urologists received training outside their home country: $60 \%$ of HIC urologists and $34 \%$ LMIC urologists. The most common countries for LMIC urologists to receive training were South Africa, Thailand and Costa Rica. HIC urologists received their training most commonly in the United Kingdom, United States or Japan (Fig. 2).

\section{Urologic conditions treated}

The most common conditions treated by surveyed urologists were BPH and urolithiasis in both HIC and LMIC (Fig. 3) A greater number of LMICs reported treating vesicovaginal fistula (VVF) $(p<0.05)$. There was greater diversity in the selected treatments for common benign urologic conditions in LMICs with an open approach being more common in LMICs (Fig. 4). The majority (61\%) of HIC urologists would treat an $8 \mathrm{~mm}$ proximal ureteral stone ureteroscopically, whereas in LMIC only $37 \%$ would perform ureteroscopy and $16 \%$ would perform open ureterotomy. A patient with a $70 \mathrm{~g}$ prostate and urinary retention would get a transurethral resection by $90 \%$ of HIC urologists, whereas only $47 \%$ of LMIC urologists would offer endoscopic resection and $36 \%$ would perform open simple prostatectomy.

Across all categories, patients in LMIC were reportedly less likely $(p<0.001)$ to get the accepted "standard" treatment for several urologic conditions as defined by the American Urological Association (AUA) guidelines statements. Figure 5 shows that patients in LMIC were approximately one Likert category less likely to get drained for an infected stone, get BCG for high-risk non-invasive bladder cancer, get a 24-h urine test for recurrent stone formers or be offered a nephron-sparing approach for a $5 \mathrm{~cm}$ renal mass.

\section{Challenges to urologic practice}

Compared to urologists in HICs, local LMIC urologists reported experiencing challenges to providing urologic care more often due to the lack of access to preoperative diagnostics, intraoperative radiology, disposables surgical supplies and implants. But areas with the greatest discrepancy between HIC and LMIC urologists were "patient's ability to pay" and "insufficient reimbursement" with LMIC urologists citing these financial challenges more frequently. Interestingly, there was not a significant difference between local LMIC and HIC rating of knowledge, surgical skill level, or OR facilities and staffing (Fig. 6).

Local LMIC and visiting LMIC urologists similarly identified the challenges due to patient's ability to pay for care and reimbursement. But in every other category, visiting LMIC 
urologists reported greater challenges related to knowledge, surgical skills, OR facilities, support staff, diagnostics, disposables and implants. For local LMIC urologists, disposables and implants were greater challenges in comparison to visiting LMIC urologist who were more likely to cite challenges in operative facilities, staff, disposables and diagnostics as the major challenges. Safe and effective anesthesia was the least often reported challenge in every group. There was no significant difference in the reported level of challenge of "patient's adherence to treatment" and "cultural beliefs" in HIC, local LMIC and visiting LMIC urologists.

Visiting LMIC urologists cited motivations for international practice was to "educate or train clinicians/surgeons in that country" (76\%) and "to provide clinical care/surgery to patients that is limited in that country" (52\%). Self-education and income generation was reported by a minority $(<20 \%)$. The majority (68\%) of visiting LMIC urologists felt confident in their ability to judge the challenges faced in the LMICs.

\section{Discussion}

Our study supports prior reports that urologists who visit LMICs to practice usually do so altruistically to teach their fellow urologists and to treat patients who may not otherwise have access to care $[5,6]$. This is in response to a clear need for more urologists in these countries. Our survey revealed interesting patterns in the background and training of urologists who volunteered to work in LMICs. We found that of the urologists surveyed, those that visited LMICs to practice were more likely to have been born in a LMIC. This may suggest that urologists who immigrated are more likely to engage in international volunteer work and supporting immigrant urologists can bolster international collaborations, which has been reported in other surgical specialties [9]. We found that local LMIC urologists are actually less likely to obtain training outside their home country compared to HIC urologist from outside the US and Europe. Local LMIC urologists who received outside training more often sought training at regional centers. This trend is likely to be multifactorial [9]. Such factors may include barriers to accessing training in the US and Europe, cost or logistics of the travel. More investigation of the barriers to training in the US and Europe for LMIC urologists is warranted.

The spectrum of diseases treated by HIC and LMIC urologists was similar in each group and previously reported surveys [10]. This should be reassuring to urologists interested in international volunteering. However, the methods of treatment of common benign conditions were heavily skewed toward more open procedures in LMICs. This trend is an opportunity for international surgical educators to focus training on minimally invasive techniques. It also represents an opportunity for trainees from HICs to observe open approaches that may no longer be performed at their home institutions. There was a trend in LMICs urologists reporting that patients were less likely to receive AUA and other international standards of care for several representative clinical scenarios, including drainage for an infected obstructed stone, a potentially life-threatening and emergent condition. International organizations may have greater impact if more effort is spent on implementing changes to achieve discrete standards in care. 
Unsurprisingly, we found LMIC urologists face challenges to providing care at greater frequency than their HIC counterparts overall. Contrary to some traditional assumptions, patient adherence and cultural beliefs did not present a greater challenge in LMICs than HICs. Our survey identified that for local LMIC urologists, the greatest barriers were financial, supporting previous commentary $[2,5,9]$. We found discrepancy between the challenges identified by the local LMIC and the visiting LMIC urologists. Visiting LMIC urologists identified challenges at much greater frequency than the local LMIC in all areas except financial and cultural. Visiting urologists who were accustomed to more wellresourced clinical settings in their home practice may have found the challenges of practicing without these resources greater.

Despite the biases toward large referral and academic hospitals, our survey identified significant challenges to providing urologic care. Broad categorization was useful for comparison at the level of income-grouping to highlight how experience and opinion can vary between practice setting and the context of the practitioner. However, it is also interesting to note that there were areas that were equally challenging to the practice of urology regardless of income-grouping, such as "patient's adherence" and "cultural beliefs." Less than two-thirds of visiting urologists felt fully comfortable evaluating the challenges to urologic care in LMIC which speaks to the humility of respondents working in these areas. This should also encourage international partners to continue open and honest dialogue to clarify the local problems that need to be addressed to improve overall patient care $[5,7]$.

Our survey is the largest published to date on challenges of care for international urologists and the first to compare local and visiting LMIC urologists. We obtained responses from every continent (except Antarctica), achieved representation from diverse regions of the globe from multi-national organizations. However, there are notable limitations to our survey. Most importantly, our survey is not representative of the average practicing urologists in any country. Those surveyed represent a cohort that is especially interconnected globally and have academic interest in regional societies and international collaboration. Due to the method of survey distribution, these urologists are concentrated in large cities and academic centers and are more likely to be sub-specialists and prevent us from calculating a response rate. The online format and single language in which it was published, also biased respondents to be from English-speaking countries with easy access to the internet and digital literacy. There was poor representation from large population countries including Russia, China, India, and Brazil. Our small sample size in each group limited statistical analysis such as multivariate regressions. The classification of countries into LMIC and HIC is inherently broad and obscures the important country- or even city-specific challenges in health care delivery. The local LMIC responses may not be comparing the same healthcare location as those characterized by the visiting LMIC urologists, even when describing the same country. Therefore, differences between groups may also represent differences in facilities.

\section{Conclusion}

Local needs-driven international collaboration among urologists has the potential to expand access to urologic care in low-resourced settings and refine the subspecialized care at centers 
of excellence. But there can be a disconnect between the needs identified by local and visiting urologists. Further research should be done to determine context-specific needs of international partners and interventions may need to expand beyond training support. Expanding efforts to include health care systems that target standards of care offers significant potential for improving urologic care worldwide.

\section{References}

1. Meara JG, Leather AJ, Hagander L et al. (2015) Global Surgery 2030: evidence and solutions for achieving health, welfare, and economic development. Lancet 386(9993):569-624 [PubMed: 25924834]

2. Patel HD, Kushner AL, Allaf ME (2013) Waiting for global access to urologic care. Eur Urol 64(2):344-345

3. Murray CJ, Vos T, Lozano R et al. (2012) Disability-adjusted life years (DALYs) for 291 diseases and injuries in 21 regions, 1990-2010: a systematic analysis for the Global Burden of Disease Study 2010. Lancet 380(9859):2197-2223 [PubMed: 23245608]

4. Patel HD, Kamara TB, Kushner AL et al. (2014) Gross hematuria and urinary retention among men from a nationally representative survey in Sierra Leone. Urology 83(6):1273-1278 [PubMed: 24726148]

5. Badlani G (2017) International volunteerism and global responsibility. Transl Androl Urol 6(2):258263 [PubMed: 28540233]

6. Konety BR (2013) Of albert Schweitzer and global urology. J Urol 189(2):411-412 [PubMed: 23201313]

7. Jalloh M, Wood JP, Fredley M et al. (2015) IVUmed: a nonprofit model for surgical training in lowresource countries. Ann Glob Health 81(2):260-264 [PubMed: 26088092]

8. “About Urolink". https://www.baus.org.uk/professionals/urolink/about_urolink_2017.aspx

9. Hoyler M, Finlayson SR, McClain CD, Meara JG, Hagander L (2014) Shortage of doctors, shortage of data: a review of the global surgery, obstetrics, and anesthesia workforce literature. World J Surg 38(2):269-280. 10.1007/s00268-013-2324-y [PubMed: 24218153]

10. Campain NJ, MacDonagh RP, Mteta KA, McGrath JS, BAUS Urolink (2015) Global surgeryhow much of the burden is urological? BJU Int 116(3):314-316 [PubMed: 25943037] 

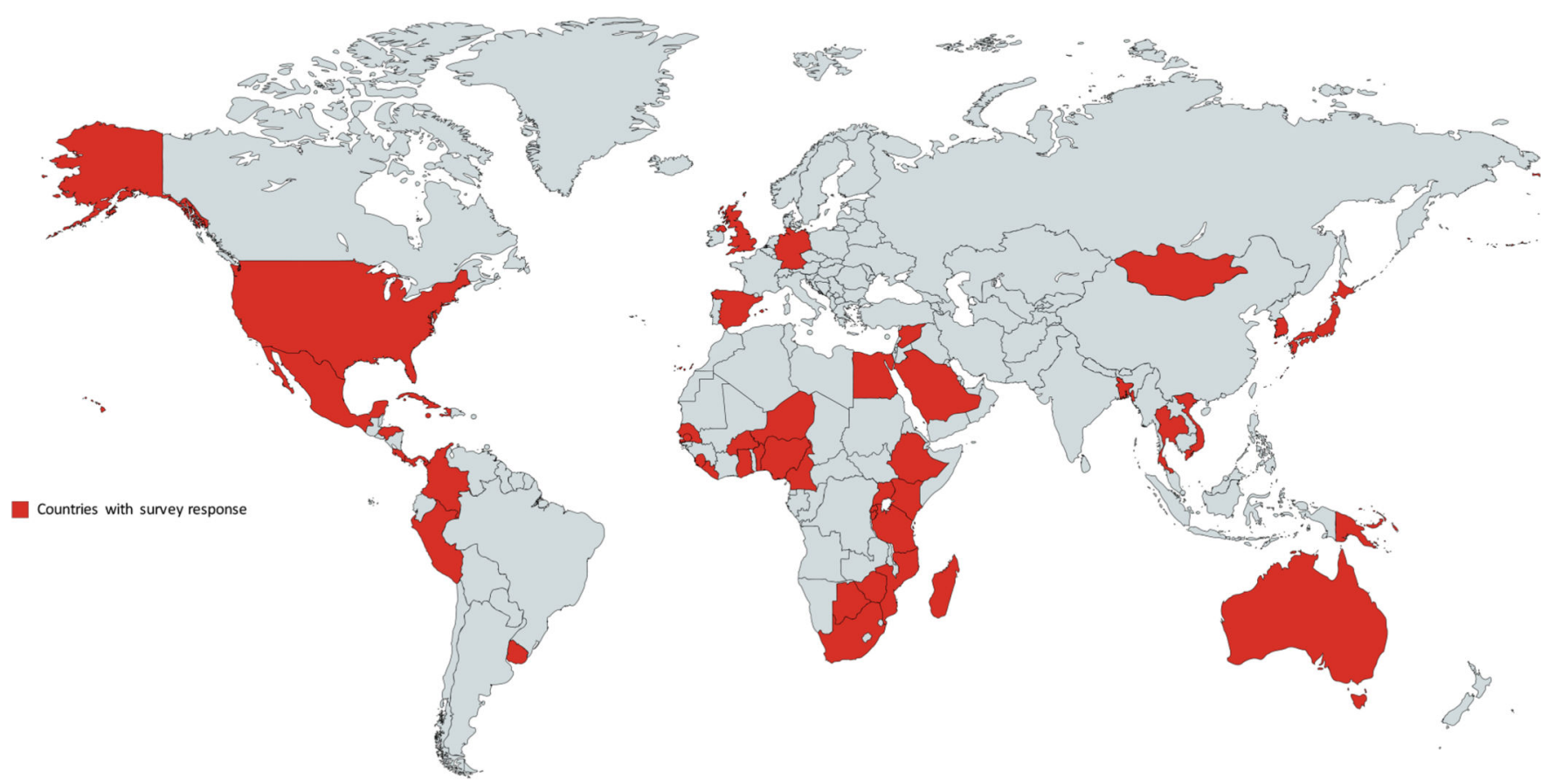

Figure 1.

Map of All Respondent Countries 


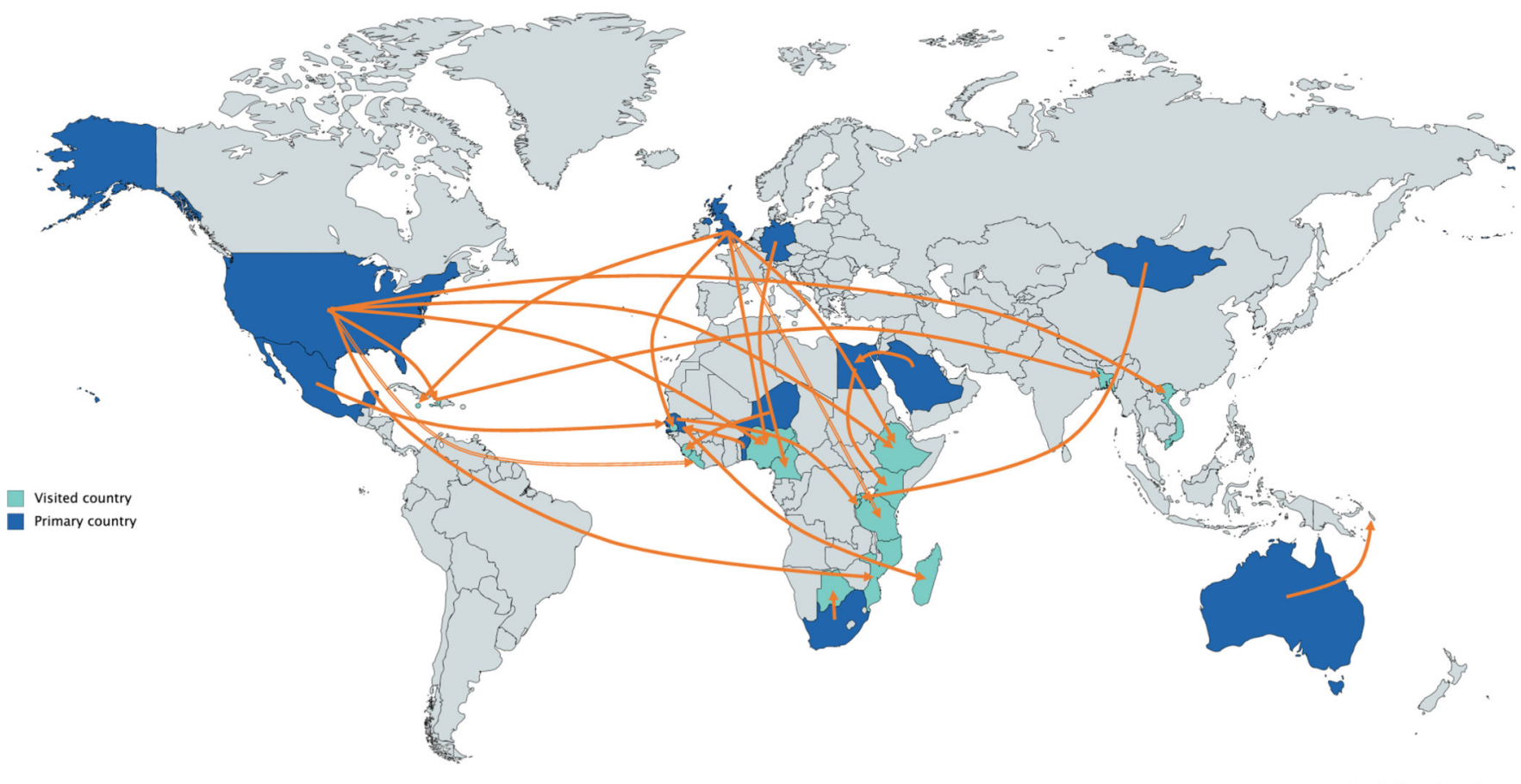

Figure 2.

Map of International Travel by Visiting LMIC Urologists 
$100 \%$

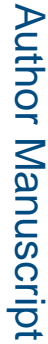

Figure 3.

Most Commonly Treated Conditions by Income Group

World J Urol. Author manuscript; available in PMC 2021 November 01 


\section{Most common treatment for an $8 \mathrm{~mm}$ proximal ureteral stone}
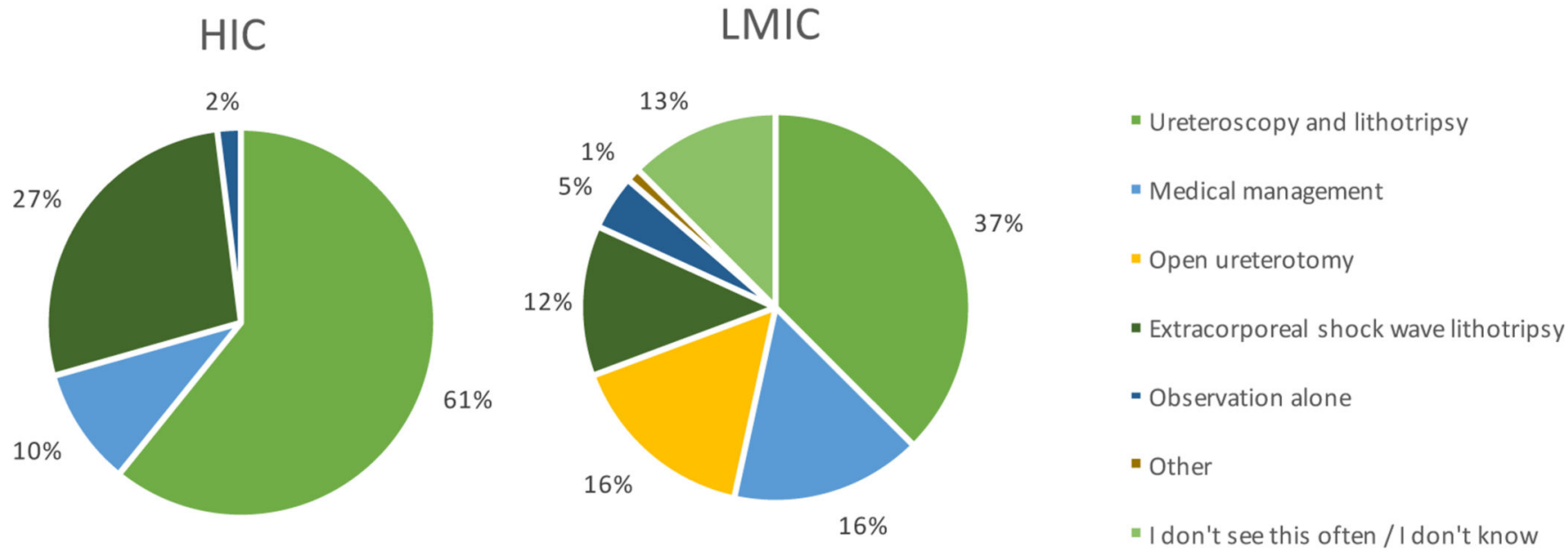

- Ureteroscopy and lithotripsy

- Medical management

- Open ureterotomy

- Extracorporeal shock wave lithotripsy

- Observation alone

- Other

" I don't see this often / I don't know

\section{Most common treatment for a 70gm obstructing prostate $\mathrm{HIC}$}
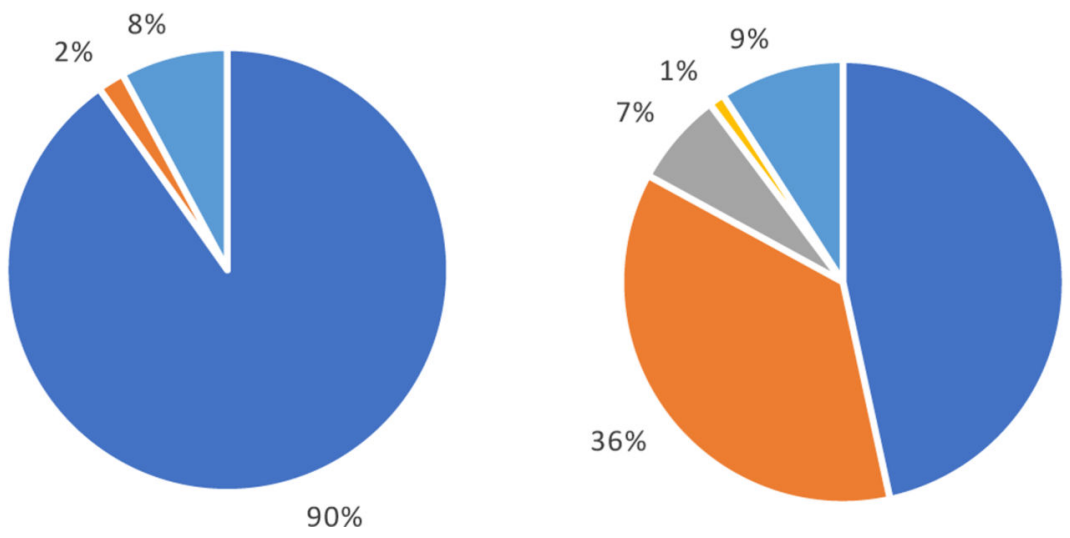

- Transurethral resection of the prostate

- Open simple prostatectomy

- Chronic indwelling urethral catheter

- Chronic suprapubic tube placement

- I don't see this often / I don't know

Figure 4.

Treatment for Common Benign Urologic Conditions 


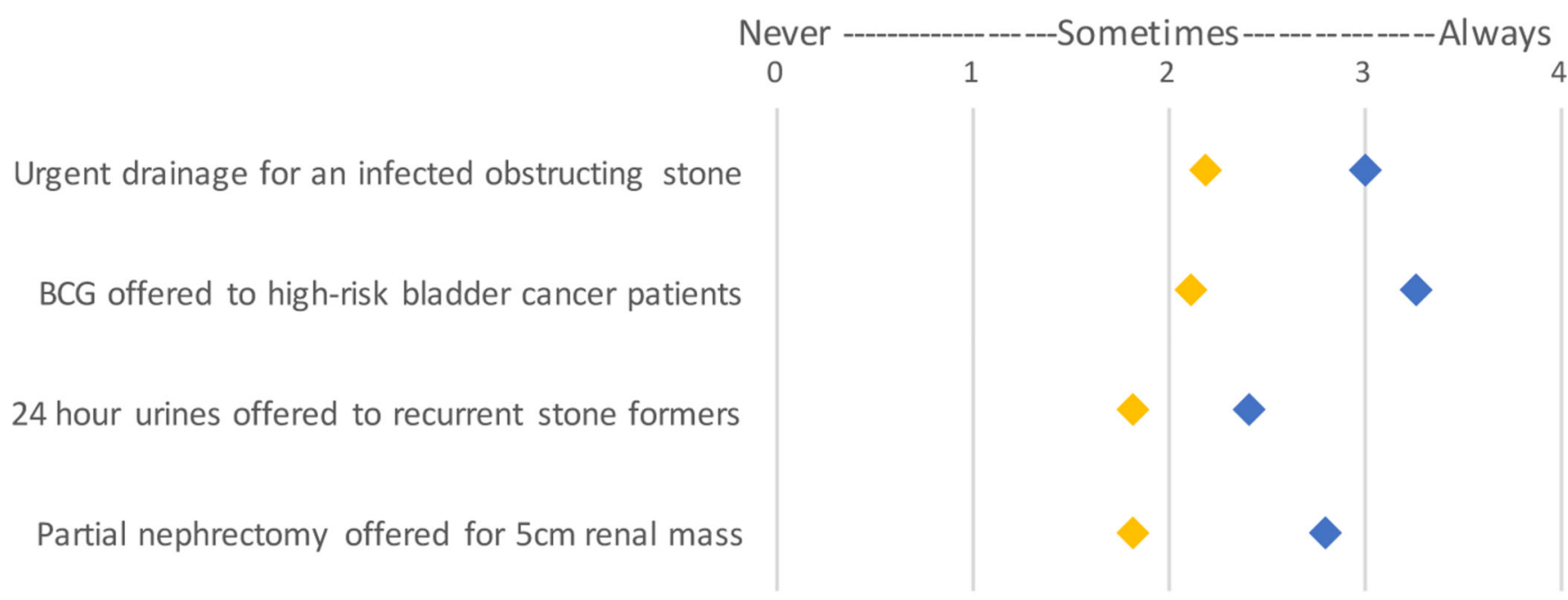

HICS $(n=35) \quad$ Locals in LMIC $(n=54)$

Figure 5.

Likelihood of Meeting AUA "Standards" of Care 


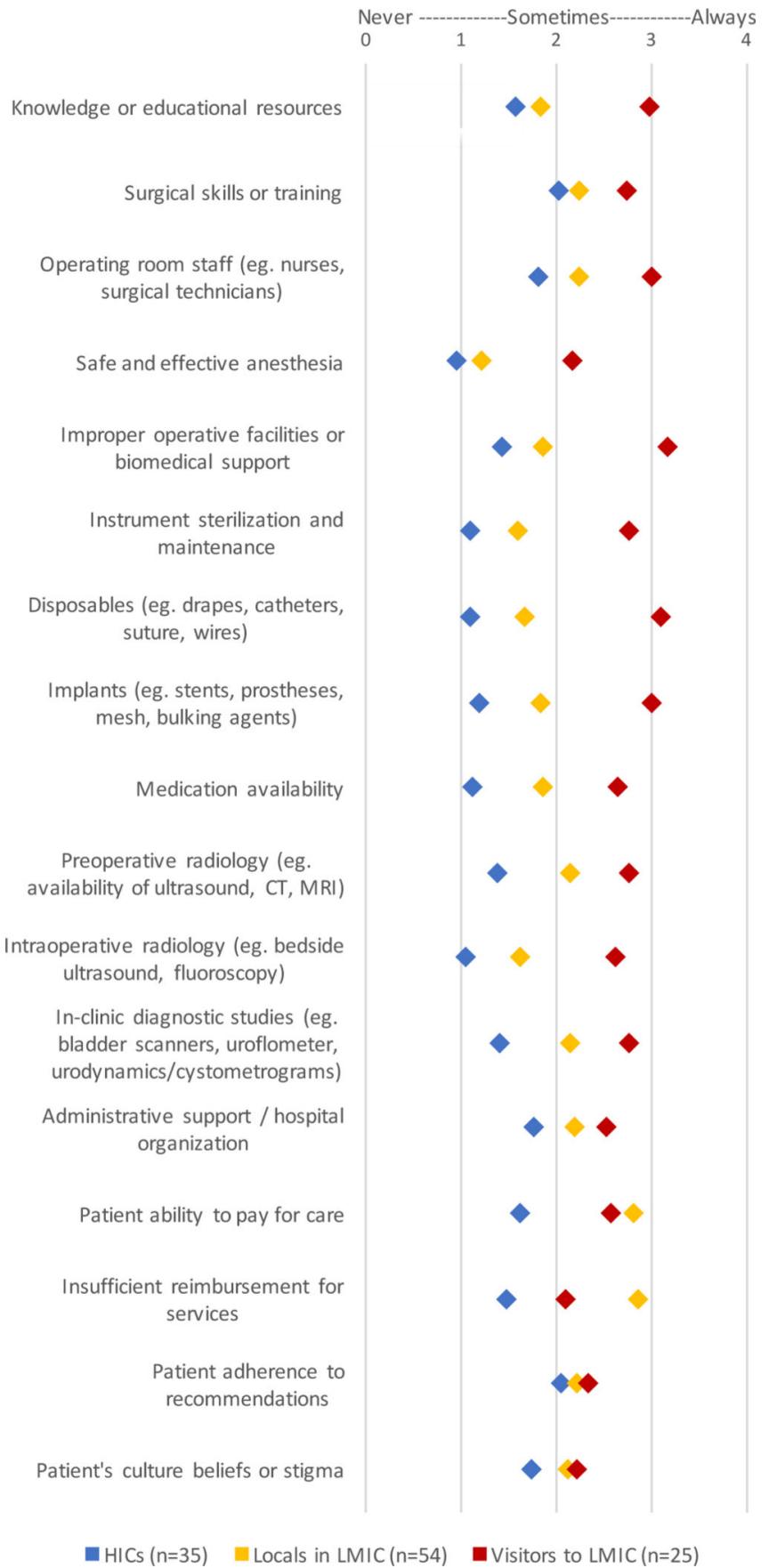

Figure 6.

Challenges to Urologic Care Identified by Income Group 
Table 1.

Demographics and Training of Surveyed Urologists

\begin{tabular}{|c|c|c|c|}
\hline Category & HIC only (n=35) & Local LMIC (n=54) & Visiting LMIC $(n=25)$ \\
\hline \multicolumn{4}{|l|}{ Age } \\
\hline Average (years) & 48.1 & 46.0 & 49.1 \\
\hline \multicolumn{4}{|l|}{ Sex } \\
\hline Female & 5 & 3 & 5 \\
\hline Male & 30 & 51 & 20 \\
\hline \multicolumn{4}{|l|}{ Income group of birth country } \\
\hline $\mathrm{HIC}$ & 30 & 1 & 14 \\
\hline LMIC & 5 & 53 & 11 \\
\hline \multicolumn{4}{|l|}{ Years of general surgery training } \\
\hline Average (years) & 2.6 & 2.0 & 2.1 \\
\hline \multicolumn{4}{|c|}{ Years of urology training (including fellowships) } \\
\hline Average (years) & 5.4 & 4.1 & 4.8 \\
\hline \multicolumn{4}{|c|}{ Urology training outside home country } \\
\hline Yes $(\%)$ & $21(60)$ & $20(37)$ & $7(28)$ \\
\hline \multicolumn{4}{|c|}{ In which country did you receive your urology training? } \\
\hline Country (n) & United Kingdom (6) & South Africa (16) & United States (12) \\
\hline & Japan (6) & Thailand (9) & United Kingdom (5) \\
\hline & Saudi Arabia (5) & Costa Rica (3) & Australia \\
\hline & Australia (5) & France (2) & Benin \\
\hline & Canada (4) & Haiti (2) & Egypt \\
\hline & United States (3) & Nigeria (2) & Germany \\
\hline & South Korea (2) & Panama (2) & Mexico \\
\hline & Germany & Argentina & Senegal \\
\hline & Mexico & Benin & South Africa \\
\hline & Pakistan & Chile & Trinidad and Tobago \\
\hline & Uruguay & Colombia & \\
\hline & & Cuba & \\
\hline & & Ethiopia & \\
\hline & & Ghana & \\
\hline & & India & \\
\hline & & Jamaica & \\
\hline & & Mexico & \\
\hline & & Peru & \\
\hline & & Russia & \\
\hline & & Senegal & \\
\hline & & Syrian Arab Republic & \\
\hline & & United Kingdom & \\
\hline & & Vietnam & \\
\hline & & Zimbabwe & \\
\hline
\end{tabular}

World J Urol. Author manuscript; available in PMC 2021 November 01. 
Table 2.

Practice Characteristics of Surveyed Urologists

\begin{tabular}{|c|c|c|c|}
\hline Category & HIC only $(n=35)$ & Local LMIC $(n=54)$ & Visiting LMIC $(n=25)$ \\
\hline \multicolumn{4}{|l|}{ Years practiced as a urologist? } \\
\hline Average (years) & 18.8 & 15.6 & 20.0 \\
\hline \multicolumn{4}{|l|}{ Type of Specialty Practice } \\
\hline General urology & 12 & 35 & 5 \\
\hline Endourology / Kidney stone disease & 7 & 5 & 3 \\
\hline Urologic oncology & 5 & 5 & 3 \\
\hline Pediatric urology & 4 & 6 & 7 \\
\hline Reconstruction / Trauma & 3 & 1 & 4 \\
\hline Female urology & 2 & 0 & 3 \\
\hline General surgery and urology mix & 1 & 1 & 0 \\
\hline Sexual and reproductive health & 1 & 1 & 0 \\
\hline \multicolumn{4}{|l|}{ Primary Practice Region } \\
\hline East Asia \& Pacific & 17 & 10 & 2 \\
\hline Europe \& Central Asia & 2 & 0 & 7 \\
\hline Latin America \& Caribbean & 3 & 17 & 2 \\
\hline Middle East \& North Africa & 12 & 1 & 2 \\
\hline North America & 1 & 0 & 7 \\
\hline Sub-Saharan Africa & 0 & 26 & 5 \\
\hline \multicolumn{4}{|l|}{ Population in Primary Practice Area } \\
\hline$<1,000$ & 0 & 1 & 0 \\
\hline $1,000-19,999$ & 0 & 2 & 0 \\
\hline 20,000-99,999 & 1 & 3 & 1 \\
\hline $100,000-299,999$ & 2 & 3 & 4 \\
\hline $300,000-999,999$ & 6 & 5 & 8 \\
\hline $1,000,000-9,999,999$ & 20 & 37 & 10 \\
\hline$>10,000,000$ & 6 & 3 & 2 \\
\hline \multicolumn{4}{|l|}{ Primary Practice Facility Type } \\
\hline Academic referral & 21 & 29 & 7 \\
\hline Private community & 8 & 18 & 3 \\
\hline Public district & 5 & 5 & 10 \\
\hline Faith-based & 0 & 0 & 3 \\
\hline Nongovernmental-organization (NGO) & 1 & 2 & 2 \\
\hline
\end{tabular}

World J Urol. Author manuscript; available in PMC 2021 November 01. 